Research Article

\title{
Three-Dimensional-Printed Guiding Template for Unicompartmental Knee Arthroplasty
}

\author{
Fei Gu, ${ }^{1,2,3}$ Liangliang Li, ${ }^{2,4}$ Huikang Zhang, ${ }^{2,5}$ Xuxiang Li, ${ }^{1,2}$ Chen Ling, ${ }^{1,2}$ \\ Liming Wang $\mathbb{D},{ }^{1,2}$ and Qingqiang Yao $\mathbb{D}^{1,2}$ \\ ${ }^{1}$ Department of Orthopaedics, Nanjing First Hospital, Nanjing Medical University, No. 68, Changle Rd, Qinhuai District, \\ Nanjing 210006, China \\ ${ }^{2}$ Institute of Digital Medicine, Nanjing Medical University, No. 68, Changle Rd, Qinhuai District, Nanjing 210006, China \\ ${ }^{3}$ The Second People's Hospital of Lianyungang, No. 41, Hailian East Rd, Haizhou District, Lianyungang 222000, China \\ ${ }^{4}$ Department of Orthopedics, The Affiliated Jiangning Hospital with Nanjing Medical University, Nanjing 211100, China \\ ${ }^{5}$ Nanjing Clinical Nuclear Medicine Center, Nanjing First Hospital, Nanjing Medical University, No. 68, Changle Rd, \\ Qinhuai District, Nanjing 210006, China
}

Correspondence should be addressed to Liming Wang; limingwangnj@163.com and Qingqiang Yao; yaoqingqiang@gmail.com

Received 4 August 2020; Accepted 4 December 2020; Published 19 December 2020

Academic Editor: Anish R. Kadakia

Copyright (C) 2020 Fei Gu et al. This is an open access article distributed under the Creative Commons Attribution License, which permits unrestricted use, distribution, and reproduction in any medium, provided the original work is properly cited.

\begin{abstract}
Background. For unicompartmental knee arthroplasty (UKA), accurate alignment of the limb is crucial. This study is aimed at investigating the efficacy and safety of a three-dimensional printed patient-customized guiding template (3DGT) for UKA. Methods. A total of 22 patients receiving UKA were randomly divided into the 3DGT-UKA group $(n=11)$ and traditional UKA group (T-UKA group; $n=11$ ). In the 3DGT-UKA group, the line and angle of osteotomy were decided on a 3D image of the limb reconstructed from imaging data; a guiding template was then designed and printed out. The patients in the T-UKA group underwent conventional UKA. Prosthesis size, operation time, postoperative drainage, hip-knee angle (HKA), pain, and Hospital for Special Surgery (HSS) scores were recorded at day 1, week 1, month 1, and month 3 after surgery. Results. There was no significant difference in the size of prostheses between the preoperatively designed and actually used in the 3DGT-UKA group $(p>0.05)$. HKA was comparable in 3DGT-UKA and T-UKA patients. Operation time was shorter (53.6 \pm 6.4 minutes vs. $75.8 \pm 7.1$ minutes) and wound drainage was less $(93.2 \pm 3.9 \mathrm{~mL}$ vs. $85.2 \pm 3.0 \mathrm{~mL})$ in 3DGT-UKA than in T-UKA $(p<0.05)$. Hospital stay was shorter in the 3DGT-UKA group. The 3DGT-UKA group had a lower VAS score on day 1, week 1, and month 1 and a higher HSS score on week 1 and month 1 after surgery. No varus/valgus deformity or prosthesis loosening was observed in either group at the final follow-up. Conclusion. The 3D-printed patient-customized guiding template may help decrease operation time, decrease blood loss, and improve short-term clinical outcomes in patients undergoing UKA surgery.
\end{abstract}

\section{Introduction}

The prevalence of osteoarthritis (OA) of the knee has increased rapidly with the aging of the population, and it has become a major cause of pain and disability. For medial compartment knee osteoarthritis (MOA), unicompartmental knee arthroplasty (UKA) has several advantages over total knee arthroplasty (TKA), including the preservation of almost all functions of the knee joint, lower postoperative complications rate, and quicker postoperative functional recovery. However, for successful UKA, accurate alignment of the limb is crucial; even a minimal shift may decrease the lifespan of the prosthesis and lead to a need for revision surgery $[1,2]$. Currently, the balance of soft tissues, the selection of prosthesis size, the angle of osteotomy, and related lower limb alignment are mainly decided by visual assessment of 


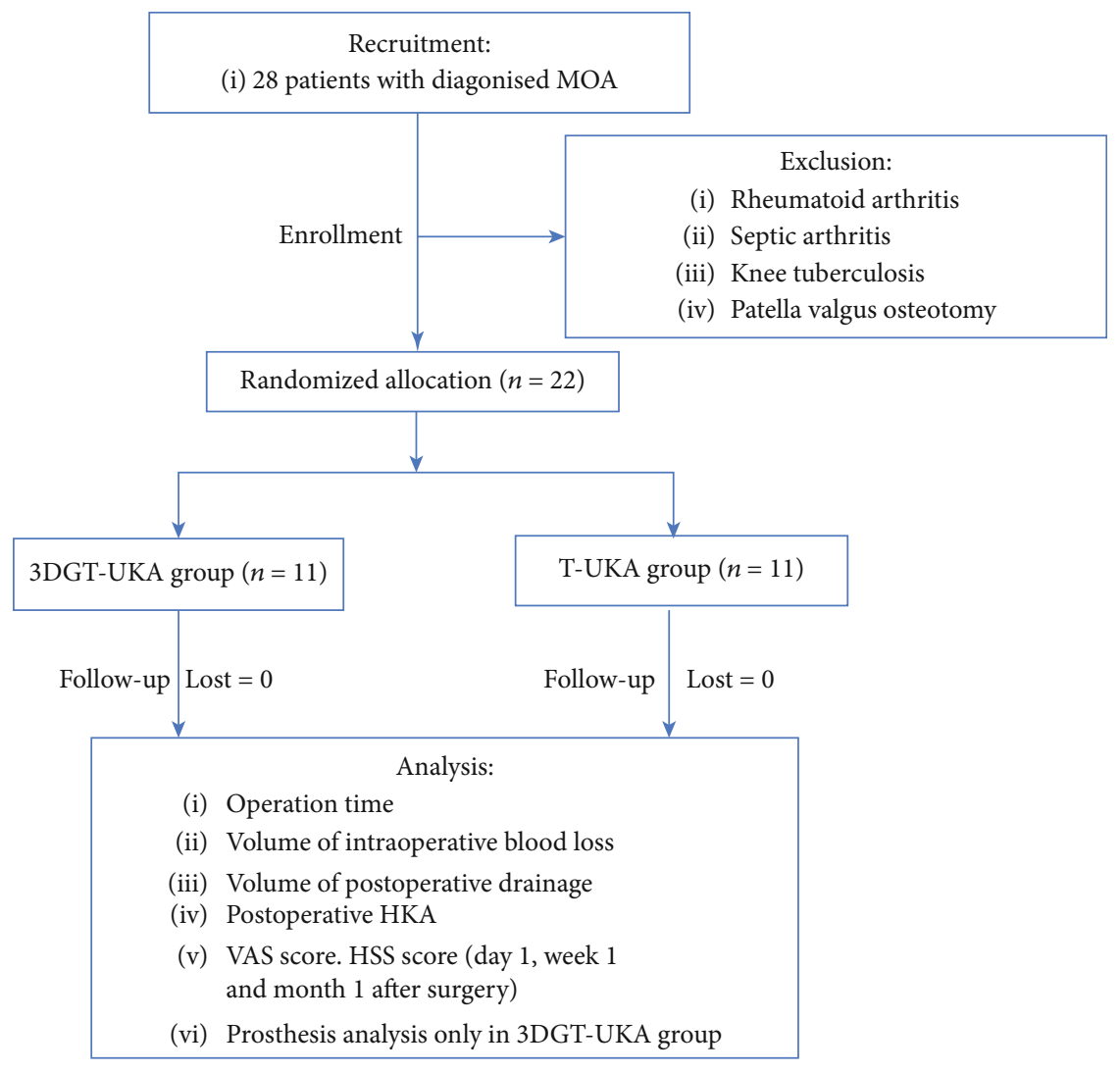

Figure 1: Consort diagram.

the surgeon. Therefore, the experience of the surgeon is one of the key factors for the reasonable performance of UKA. However, individual variations between patients and the lack of familiarity of the surgeon with the medical apparatuses might influence the final surgical effect [3].

In recent years, there has been much research on the use of three-dimensional (3D) digital image design and 3Dprinting technology to build personalized guiding templates for knee surgery. A 3D-printed individualized guiding template based on CT-MRI fusion data could greatly improve the accuracy of estimation of the angle, the amount of osteotomy, the sizes of the tibial platform, and the femoral condyle prosthesis [4-7]. The aim of this randomized clinical trial was to investigate the efficacy and safety of the use of 3Dprinted individual patient-customized guiding templates for assisting UKA.

\section{Materials and Methods}

2.1. Patients. This randomized clinical trial was performed at the Department of Orthopedics, Nanjing First Hospital, affiliated to Nanjing Medical University. MOA patients requiring UKA surgery during the period of January 2017 to December 2017 were enrolled in this study. Patients were eligible for inclusion if they had (1) a diagnosis of MOA confirmed by $\mathrm{X}$-ray and MRI, (2) intact anterior cruciate ligament, and (3) knee varus deformity that could be corrected manually. Patients with a history of rheumatoid arthritis, septic arthritis, knee tuberculosis, and patella valgus osteotomy were excluded. A total of 22 patients who met these criteria were enrolled and randomly assigned to receive either 3Dprinted patient-customized guiding template-assisted UKA (3DGT-UKA group) or traditional UKA (T-UKA group) (Figure 1).

This study was approved by the Ethics Committee of Nanjing Hospital affiliated to Nanjing Medical University. All patients gave written informed consent for participation in the study.

2.2. Preoperative Preparation. Preoperatively, all patients underwent electrocardiography, routine blood examination, and vascular ultrasonography of the lower limb. Patients with comorbidities received appropriate specialist consultations to rule out contraindications to surgery.

2.3. Design and Production of 3D-Printed Personalized Guiding Template for UKA Osteotomy. Patients in the 3DGT-UKA group first underwent a double-source 64-slice spiral CT (Siemens Sensation, Germany) full-length scan of the lower limbs and MRI scans of the knee joints. The data were imported into a workstation. CT data were processed using the Mimics 17.0 software (Materialise Ltd., Leuven, Belgium) to reconstruct a $3 \mathrm{D}$ model of the full length of the lower limbs. The MRI data were processed to obtain a 3D model of the knee joint. The two models were then merged to obtain a 3D model of the lower limbs with the cartilage lining of the knee joints also displayed. The angle between the hip and knee (HKA) and the mechanical axis of the femur 


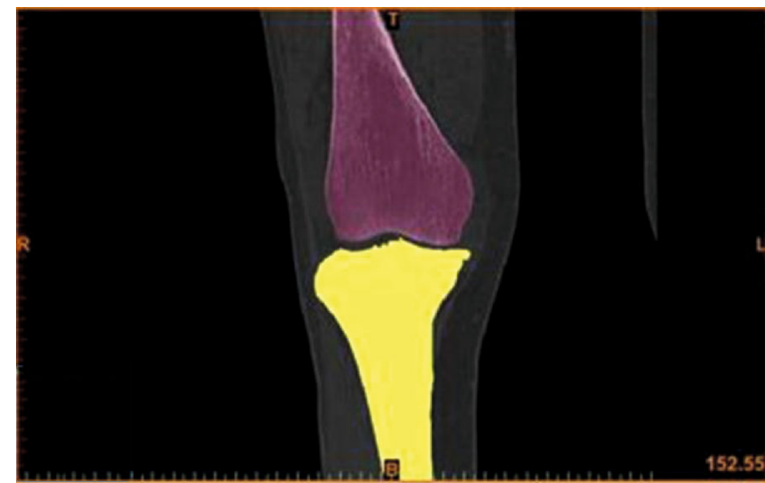

(a)

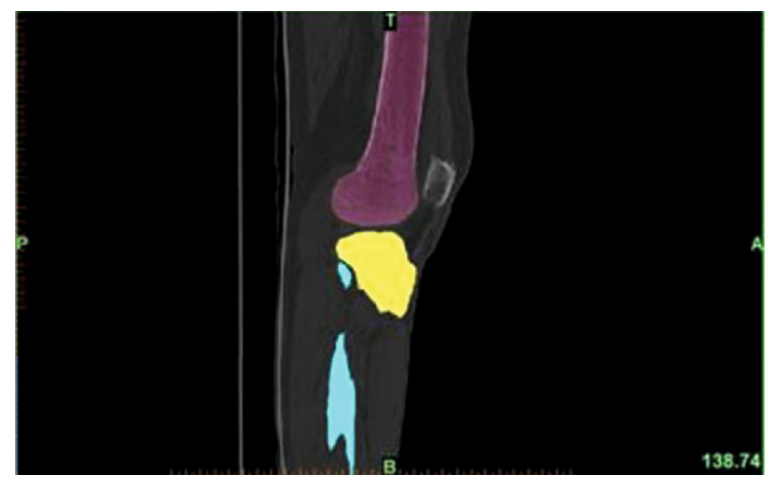

(c)

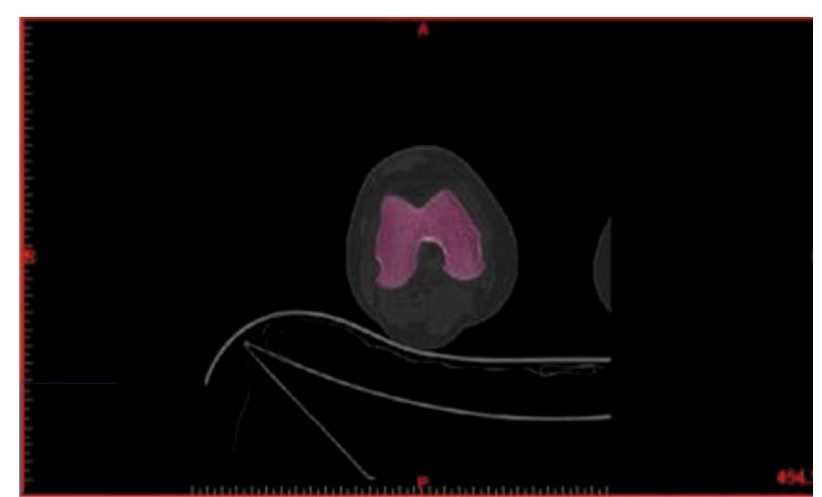

(b)

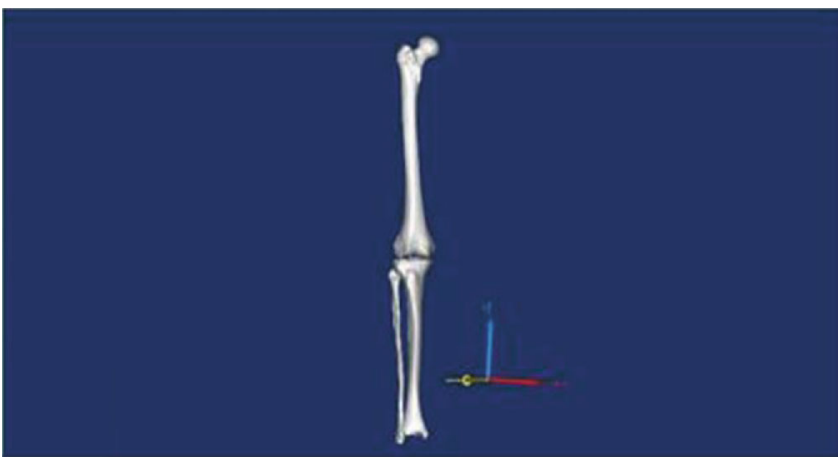

(d)

Figure 2: (a) Front view CT image of the knee joint. (b) Top view CT image of the knee joint. (c) Side view CT image of the knee joint. (d) Reconstructed three-dimensional model of a lower limb in full length.

and the tibia were measured using a computer-aided design (CAD) software (Medivi, Changzhou, China). The angle and amount of osteotomy were accurately calculated on the reconstructed $3 \mathrm{D}$ model, and the posterior oblique angle and amount of osteotomy necessary at the tibial plateau were determined. Finally, a 3D-printed customized guiding template was created for each patient. The template was sterilized with plasma before use (Figures 2 and 3 ).

2.4. Surgical Methods. The 3rd generation Oxford prostheses for UKA were used for all patients. All surgeries were performed by the same surgical team. Surgery was performed under general anesthesia. Patients in the 3DGT-UKA group were in the supine position, and a tourniquet was placed at the base of the affected limb. The hip was flexed $30^{\circ}$ and mildly abducted, with the calf hanging naturally down. Free movement of the knee joint for at least $135^{\circ}$ was ensured. With the knee flexed at $90^{\circ}$, a $6-\mathrm{cm}$ incision was made on the medial side of the knee joint, extending from the distal end of the joint line at the medial edge of the tibia up to the medial edge of the femoral muscle. The distal femoral fat pad was removed to expose the distal femur and the superior tibia. The femoral condyle, intercondylar fossa, tibial plateau epiphysis, and the soft tissue attached to the anterior side of the platform were removed. Then, the tibial 3D-printed patient-customized guiding template was carefully and closely applied on the anterior aspect of the tibial plateau, and with its guidance holes were drilled into the proximal end of the tibia. The positioning nails were inserted, and the 3D-printed patient-customized guiding template was removed. Next, the manufacturer-made matching osteotomy template (Zimmer Ltd., USA) was then positioned, and osteotomy of the tibial plateau was carried out. After the osteotomy, the dimensions of the cut tibial plateau and prosthesis were measured. The distal femur and intercondylar fossa were then exposed. The distal femoral 3D-printed guiding template was placed in position, and the drilling was carried out. The distal femoral template was removed, and the manufacturer-made posterior condylar template (Zimmer Ltd., USA) was placed for posterior condylar osteotomy. The bone bung was inserted into the distal femur for grinding. Finally, a single condylar prosthesis of the appropriate preoperatively determined size was then installed (Figure 4).

In the T-UKA group, the conventional single condylar replacement method was used. Tibial osteotomy aimed at making a downward $7^{\circ}$ of posterior tilt in the tibial plateau. The thickness of the tibial osteotomy was $2-3 \mathrm{~mm}$ less than the depth of the deepest part of the tibial wear. Osteotomy of the medial femoral condyle was performed by intramedullary positioning. After drilling the anterior angle of the anterior intercondylar fossa, the rods for intramedullary positioning were inserted. The femoral drill guide was placed, ensuring that the handle was parallel with the long axis of the tibia. The kneading surface was close to each other, and the relevant angle was adjusted. The positioning holes (diameter of $4 \mathrm{~mm}$ and $6 \mathrm{~mm}$ ) were drilled and according to the 


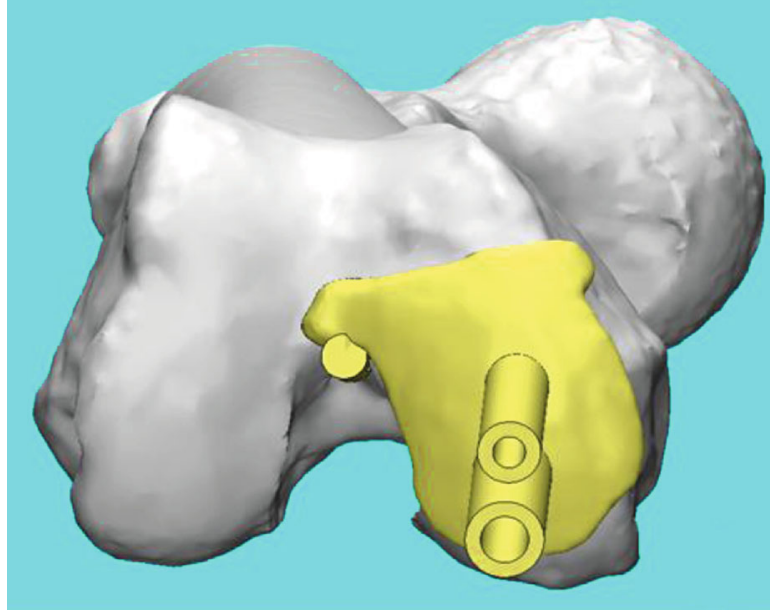

(a)

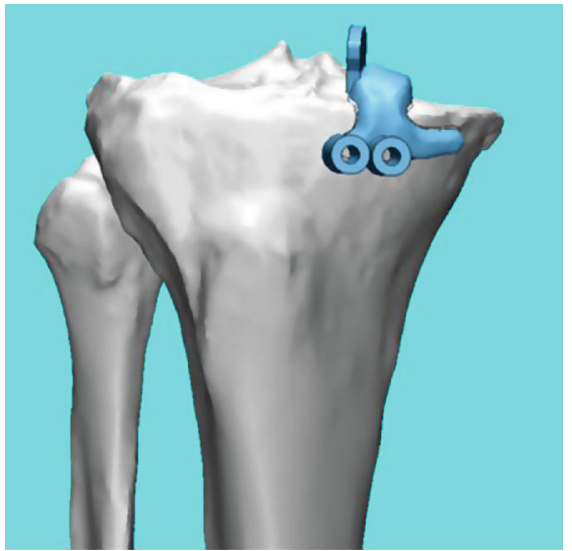

(c)

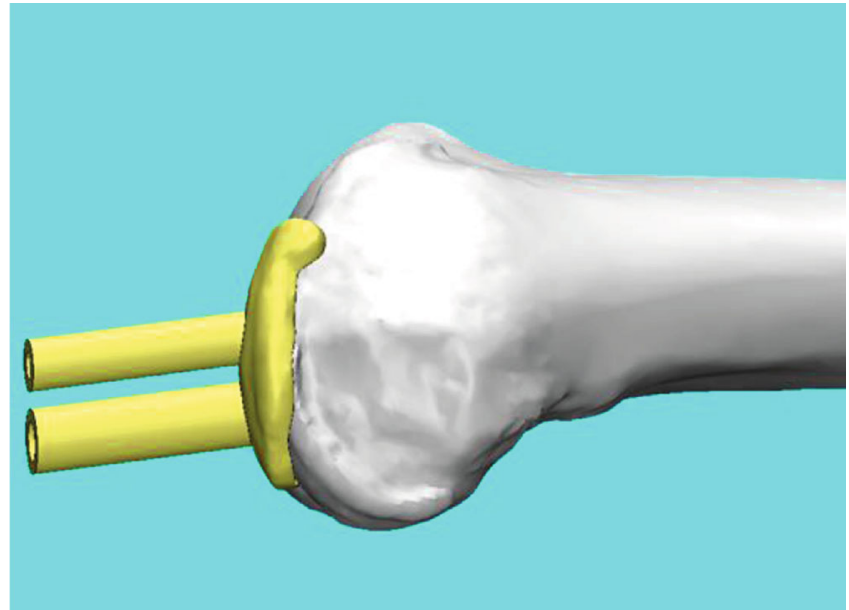

(b)

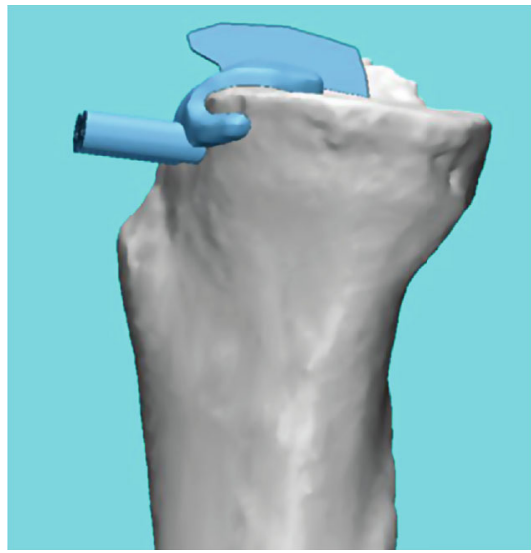

(d)

FIgURE 3: (a) Front view of the femoral 3D-printed guiding template. (b) Side view of the femoral 3D-printed guiding template. (c) Front view of the tibial 3D-printed guiding template. (d) Side view of the tibial 3D-printed guiding template.

position of them; the posterior femoral condyle was cut and ground. Prostheses of appropriate sizes were then installed into the tibia and femur.

Drainage tubes were placed in both groups after surgery [8-10]. All patients routinely received antibiotics and analgesics, as well as low-molecular-weight heparin as an anticoagulant therapy. Passive exercises were started 24 hours after surgery. The drainage tube was removed 48 hours after surgery. Patients were encouraged to walk with the help of a walking assistance device and to perform stretching and contracting exercise of quadriceps. A fulllength radiograph of the affected lower limb and a positive lateral radiograph of the knee joint were examined at 2 days postoperation, and the HKA deviation was obtained (Figure 5). Sutures were removed 2 weeks after surgery. The VAS scores on day 1 , week 1 , month 1 , and month 3 after surgery, and the Hospital for Special Surgery (HSS) scores on week 1, month 1, and month 3 after surgery, were recorded.

2.5. Postoperative Evaluation. An outpatient follow-up for three months was conducted to evaluate the recovery of the patients. And all patients involved in this study completed the postoperative follow-up. The sizes of the preoperatively designed theoretical prosthesis and that of the actual prosthesis used during the operation in the 3DGT-UKA group were compared. The operation time, intraoperative blood loss, postoperative drainage, and postoperative HKA were compared between the two groups. VAS score on day 1, week 1, month 1, and month 3 after surgery and HSS score on week 1 , month 1 , and month 3 were also compared between the two groups. Because the operation time of patients in both groups was within the set time (90 minutes) of a lower extremity tourniquet, the volume of postoperative drainage was recorded and calculated as the volume of blood loss in the current study.

2.6. Statistical Analysis. Statistical analysis was performed using the SPSS 22.0 statistical software (IBM Corp., Armonk, NY, USA). The rank-sum test was performed for ranked data in the two groups. The $t$-test was used for the analysis measurement data and the chi-squared test for enumeration data. Statistical significance was at $p \leq 0.05$. 


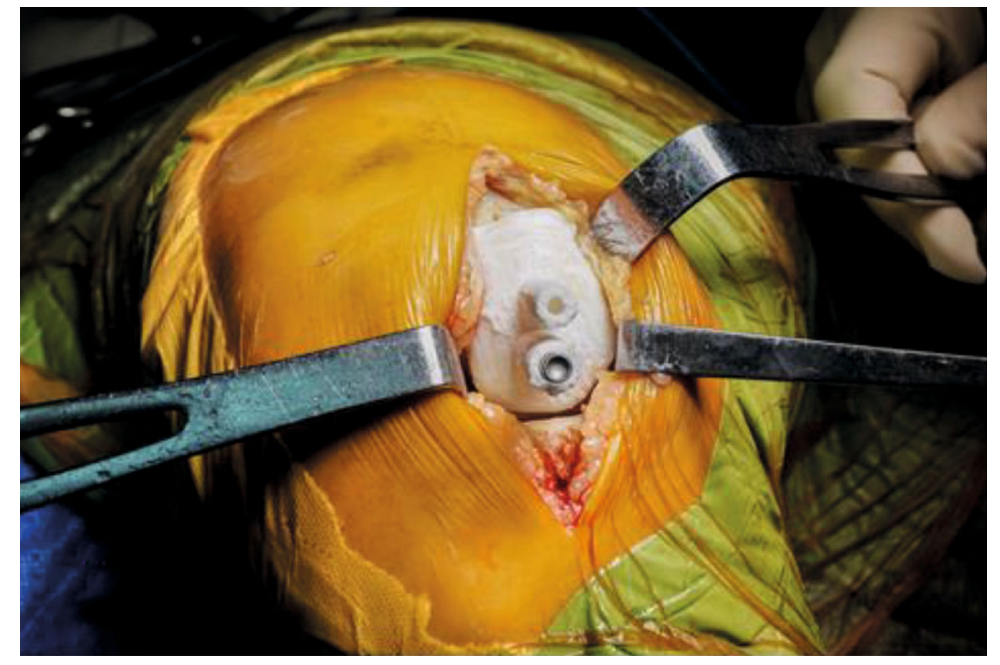

(a)

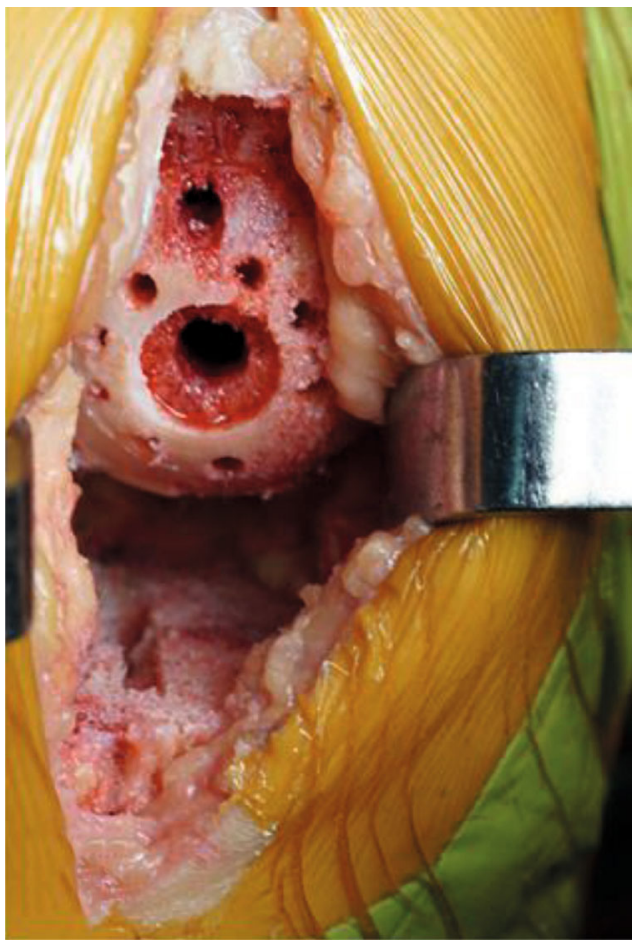

(c)

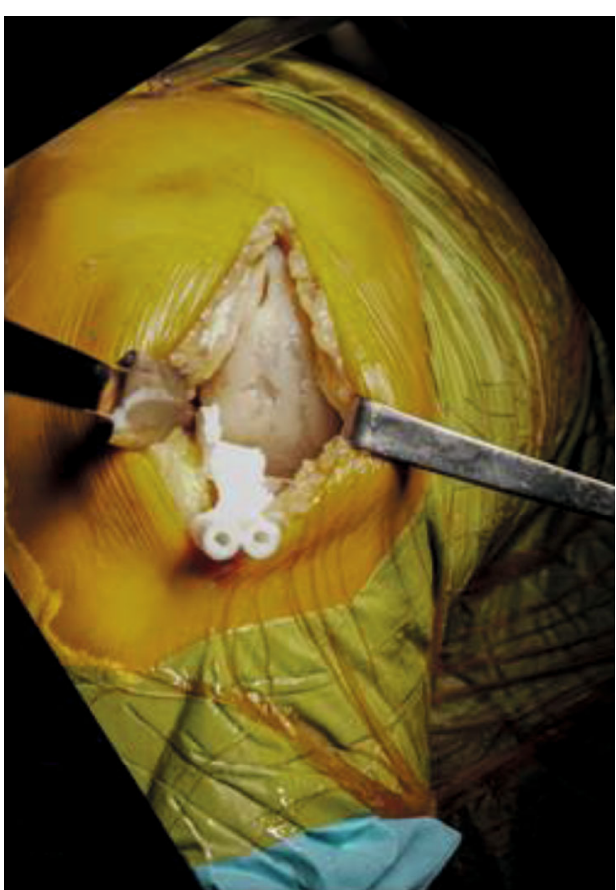

(b)

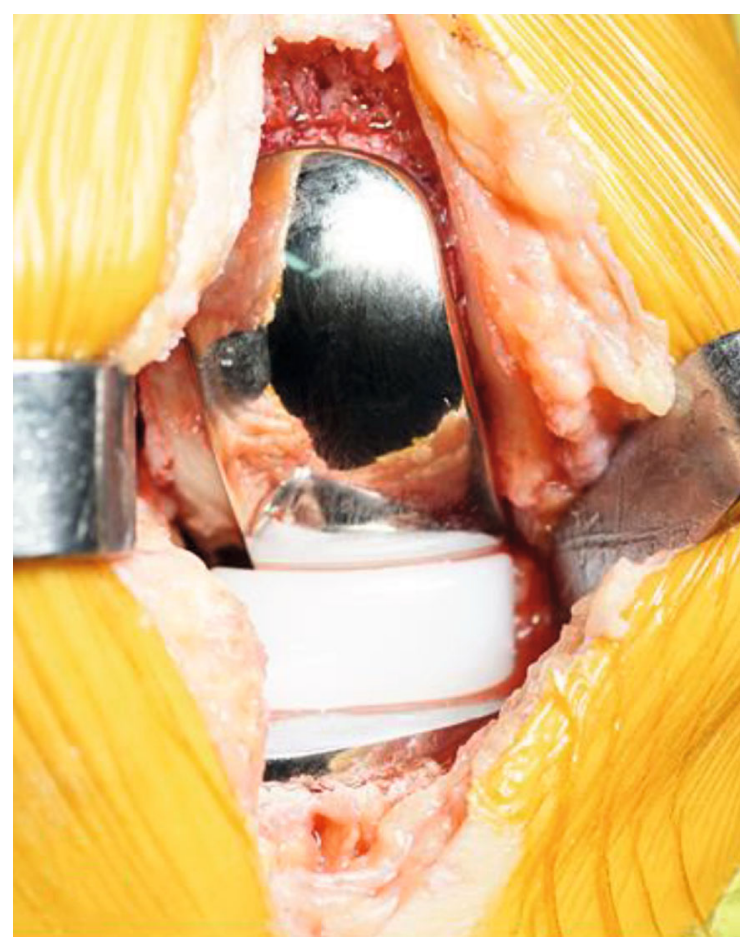

(d)

FIgURE 4: (a) Intraoperative placement of the femoral condyle 3D-printed guiding template. (b) Intraoperative placement of the tibial plateau 3D-printed guiding template. (c) Images of the femoral and tibial osteotomy. (d) Images of the installed prosthesis.

\section{Results}

A total of 22 patients ( 8 males, 14 females) with mean age $60.8 \pm 4.8$ years (age range, 52-67 years) were enrolled and randomized to receive either $3 \mathrm{DGT}-\mathrm{UKA}(n=11)$ or $\mathrm{T}$ -
UKA ( $n=11)$. The gender ratio, mean age, mean height, preoperative HSS score, and prevalence of comorbidities were comparable in the two groups (Table 1). In the 3DGT-UKA group, no significant difference was seen between the sizes of actual prosthesis used and preoperative theoretical prosthesis 


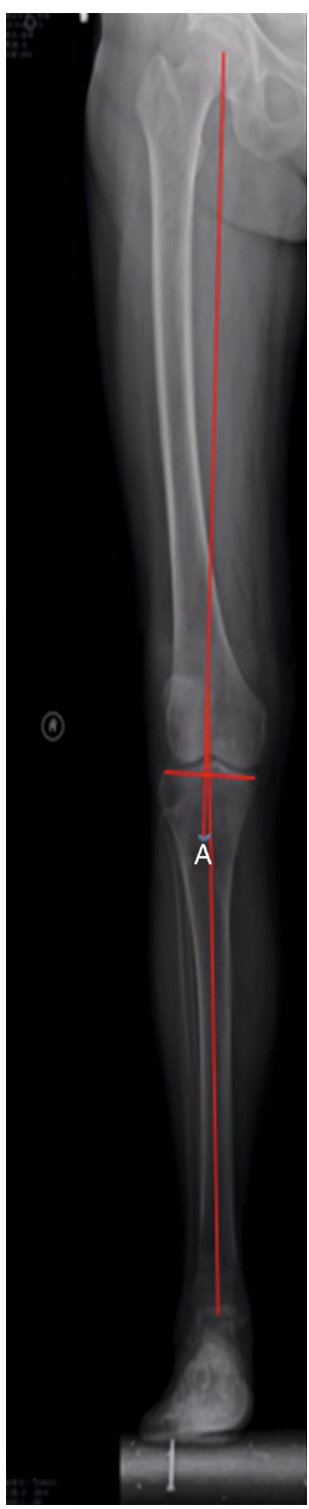

(a)

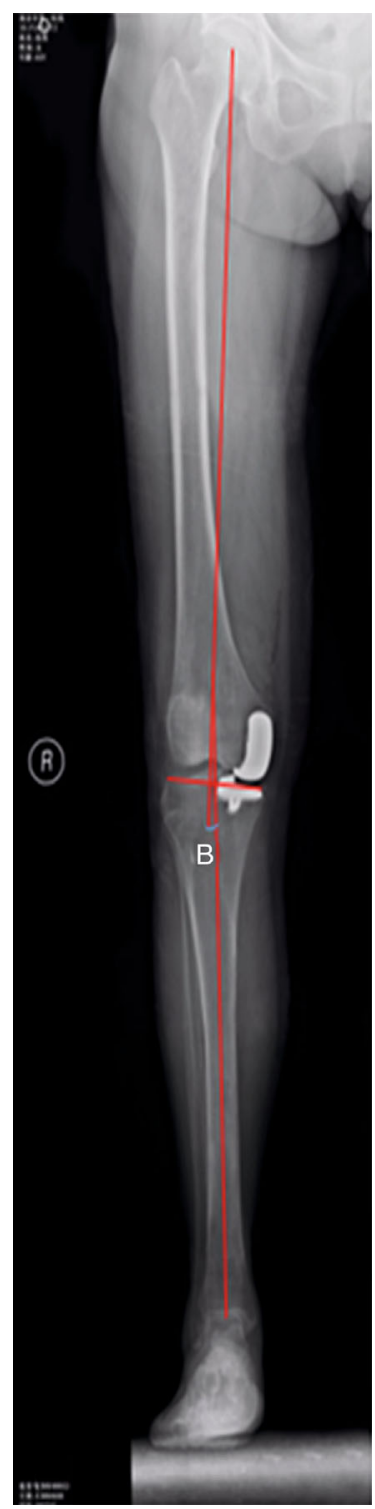

(b)

Figure 5: (a) Preoperative lower limb line (angle between the hip and knee $(\mathrm{A})=3.5^{\circ}$ ). (b) Postoperative lower limb line (angle between the hip and knee $(\mathrm{B})=2.4^{\circ}$ ).

model ( $p>0.05$; Table 2). There was no significant difference between the two groups in postoperative HKA deviation $(p>0.05$; Table 3$)$. Operation time was significantly shorter in the 3DGT-UKA group than in the T-UKA group $(53.6 \pm 6.4$ minutes vs. $75.8 \pm 7.1$ minutes; $p<0.05)$. The volume of postoperative drainage was significantly lower in the 3DGT-UKA group than in the T-UKA group $(67.5 \pm 3.9 \mathrm{~mL}$ vs. $93.2 \pm 3.0 \mathrm{~mL} ; p<0.05)$. The mean hospitalization time was longer in the T-UKA group than in the 3DGT-UKA group. The VAS scores on day 1, week 1, and month 1 after surgery were significantly lower in the 3DGT-UKA group than in the T-UKA group $(p<0.05)$. And the HSS scores on week 1 and month 1 after surgery were higher in the 3DGTUKA group than in the T-UKA group $(p<0.05)$. The HSS scores on month 3 after surgery were comparable between the two groups ( $p>0.05$; Tables 4 and 5).

\section{Discussion}

In theory, for OA of the knee with only medial compartment lesions, UKA may be a reasonable candidate by the preservation of the anterior-posterior cruciate ligaments. This means that most functions of the knee joint may be retained after UKA surgery. Previous studies proved that UKA showed significant advantages in symptom relief, knee function recovery, and postoperative complications, compared to TKA [8-11]. However, the long-term studies showed that the survival of prostheses in UKA was significantly lower than TKA, which may be caused by the inadequate surgical exposure due to the small incision used in UKA, large errors in intraoperative extracorporeal positioning of the lower extremity force line, and intramedullary positioning of the femoral condyle [12]. It has 


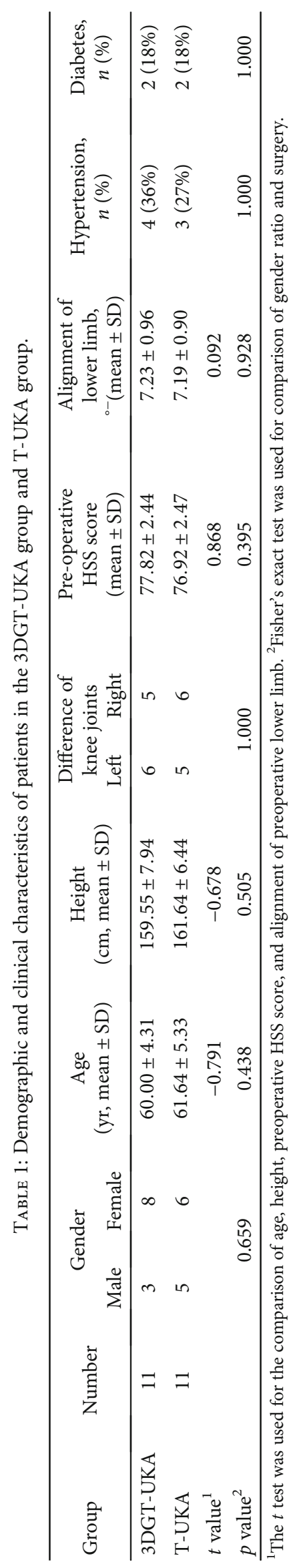


TABLE 2: Actual and theoretical values of surgical prosthesis models in the 3DGT-UKA group.

\begin{tabular}{|c|c|c|c|c|c|c|}
\hline & \multicolumn{2}{|c|}{$\begin{array}{l}\text { 3DGT-UKA femoral } \\
\text { prosthesis model }\end{array}$} & \multicolumn{4}{|c|}{ 3DGT-UKA tibial prosthesis model } \\
\hline & $M^{1}$ & S & $\mathrm{C}$ & B & A & $\mathrm{AA}$ \\
\hline Theoretical value & 1 & 10 & 0 & 4 & 5 & 2 \\
\hline Actual value & 2 & 9 & 1 & 4 & 4 & 2 \\
\hline Test statistics & 0.29 & 0.03 & 0.96 & 0 & 0.08 & 0 \\
\hline$t$ value/ $\chi^{2}$ value & 0.588 & 0.867 & 0.328 & 1.000 & 0.779 & 1.000 \\
\hline$p$ value & $>0.05$ & $>0.05$ & $>0.05$ & $>0.05$ & $>0.05$ & $>0.05$ \\
\hline
\end{tabular}

$\mathrm{M}, \mathrm{S}, \mathrm{C}, \mathrm{B}, \mathrm{A}$, and AA represent the prosthesis model of the femoral and tibial.

TABLE 3: Comparison of HKA deviation in the 3DGT-UKA group and T-UKA group.

\begin{tabular}{lcccc}
\hline Group & Number & $> \pm 2^{\circ}$ & $\begin{array}{r}\text { HKA deviation } \\
\pm 1^{\circ} \text { to } \pm 2^{\circ}\end{array}$ & $< \pm 1^{\circ}$ \\
\hline 3DGT-UKA & 11 & 3 & 5 & 3 \\
T-UKA & 11 & 4 & 4 & 3 \\
$Z$ value $^{1}$ & & & -0.28 & \\
$p$ value & & \multicolumn{3}{c}{0.78} \\
\hline
\end{tabular}

${ }^{1}$ Mann-Whitney $U$ test.

been confirmed that a slight error in the alignment of the lower limb line and a lower extremity varus increases the need for future revision surgery in patients receiving UKA. Meanwhile, postoperative valgus can significantly increase lateral compartment stress and lead to accelerated cartilage degeneration in the lateral compartment of the knee joint [13]. Therefore, in UKA patients, detailed preoperative planning and precise positioning of the intraoperative prosthesis is crucial.

Routine analysis of radiography, CT, and MRI images of the knee joint is difficult to accurately obtain the $3 \mathrm{D}$ structure of the lower extremities, and it is also difficult to quantitatively show the cartilage wear of the medial compartment. It looks like the measurement error cannot be avoided during the operation because the positioning of the tibial plateau in T-UKA patients depends on the extramedullary positioning rods, which is subjective. Furthermore, deviations in the intramedullary positioning of the distal femoral condyle and any other errors in the direction of the positioning rod may lead to impingement or unsatisfactory meniscus trajectory, as well as prolonged operation time, increased blood loss, and the risk of intraoperative fat embolism. Ma et al. studied the positional differences of unicompartmental prostheses caused by the deviation of the intramedullary positioning entrance points in the femurs of 20 corpses and concluded that the intramedullary positioning rods could not accurately indicate the actual anatomical axis of the femur, which usually leads to a deviation with $3.2^{\circ}$ of deflection and $2.5^{\circ}$ of valgus [14]. Baldini et al. found that $10 \%-20 \%$ of patients who received T-UKA had a significant deviation of the femoral force line on the prosthetic side from the normal [15]. Therefore, we felt that a $3 \mathrm{D}$-printed individualized guiding template based on quantitative anatomical data and accurate preoperative CAD measurement results would be helpful in the selection of the appropriate osteotomy angle and amount. In addition, there is no need for opening of the femoral bone marrow during femoral osteotomy with 3DGT-UKA, which helps reduce the surgical exposure time, postoperative blood loss, and amount of intraoperative instrumentation.

A 3D-printed guiding template based on CT data alone is difficult to achieve good intraoperative fitting of the guiding template due to it does not take into consideration the cartilage tissue influence, which has been shown to be responsible for the decrease in the accuracy of the 3D-printed guiding template [16]. Therefore, in this present study, the CT and MRI data were merged to reconstruct the $3 \mathrm{D}$ anatomy of the knee joint, which allowed accurate calculation of the lower limb force line, angle, and osteotomy amount. This method can help us design an accurate 3D-printed patient-customized guiding template during UKA surgery. Due to the refined and minimally invasive design of the third-generation Oxford knee prosthesis for UKA, the flexible meniscus pad also reduces the risk of impingement and rotation of the prosthesis. Therefore, the design of the $3 \mathrm{D}$-printed guiding template in this study was based on the third-generation Oxford UKA tool kit. We found there was no significant statistical difference in dimensions between the preoperatively designed theoretical prosthesis and the actual prosthesis used in the 3DGT-UKA group. There was also no significant difference in HKA between the 3DGT-UKA and T-UKA groups, indicating the accuracy of the 3DGT-UKA surgery $[17,18]$.

Compared to the 3DGT-UKA group, which prosthesis selection is based on accurate preoperative measurements of the reconstructed $3 \mathrm{D}$ knee joint, leading to less errors and rapid surgery completion $[19,20]$, the T-UKA group select the prosthesis model based on preoperative radiographic measurements, which is subjective and may cause large errors. Therefore, repeated measurements are necessary during surgery to select the appropriate size. In addition, the 3D-printed guiding template easily and accurately locates the surgical osteotomy tool without slotting, which also contributes to the decrease in operation time and postoperative drainage volume. Also, we found the 3Dprinted guiding template can help to reduce surgical trauma during UKA surgery and the duration of 
TABLE 4: Comparison of surgical parameters and outcomes between the 3DGT-UKA group and the T-UKA group.

\begin{tabular}{lcccc}
\hline Group & Number & $\begin{array}{c}\text { Operation time } \\
(\mathrm{min}, \text { mean } \pm \mathrm{SD})\end{array}$ & $\begin{array}{c}\text { Volume of postoperative drainage in } \\
\text { 48 hours }(\mathrm{mL}, \text { mean } \pm \mathrm{SD})\end{array}$ & $\begin{array}{c}\text { Postoperative HKA } \\
\left({ }^{\circ}, \mathrm{mean} \pm \mathrm{SD}\right)\end{array}$ \\
\hline 3DGT-UKA & 11 & $75.82 \pm 6.91$ & $85.18 \pm 2.96$ & $1.74 \pm 0.78$ \\
T-UKA & 11 & $67.64 \pm 6.41$ & $67.55 \pm 3.86$ & $1.81 \pm 0.67$ \\
$t$ value & & 2.879 & 12.031 & -0.205 \\
$p$ value & & 0.009 & 0.000 & 0.840 \\
\hline
\end{tabular}

TABLE 5: Comparison of postoperative VAS score and HSS score between the 3DGT-UKA group and the T-UKA group.

\begin{tabular}{|c|c|c|c|c|c|c|c|}
\hline \multirow{2}{*}{ Group } & \multicolumn{4}{|c|}{ Postoperative VAS score $($ mean \pm SD) } & \multicolumn{3}{|c|}{ Postoperative HSS score (mean \pm SD) } \\
\hline & Day 1 & Week 1 & Month 1 & Month 3 & Week 1 & Month 1 & Month 3 \\
\hline 3DGT-UKA & $4.64 \pm 0.92$ & $2.00 \pm 0.77$ & $0.82 \pm 0.60$ & $0.46 \pm 0.52$ & $80.09 \pm 5.87$ & $95.18 \pm 1.40$ & $96.46 \pm 1.44$ \\
\hline T-UKA & $7.64 \pm 0.92$ & $5.70 \pm 0.90$ & $2.46 \pm 0.82$ & $0.27 \pm 0.47$ & $70.10 \pm 5.87$ & $93.73 \pm 1.27$ & $96.46 \pm 1.21$ \\
\hline$t$ value & 7.611 & 10.381 & 5.331 & 0.861 & 3.993 & 2.549 & 0.000 \\
\hline$p$ value & $<0.001$ & $<0.001$ & $<0.001$ & 0.146 & 0.001 & 0.019 & 0.482 \\
\hline
\end{tabular}

tourniquet application, which is probably related to the lower VAS score on day 1 and week 1 and higher HSS score on week 1 and month 1 after surgery in the 3DGT-UKA group.

There are also some disadvantages with 3DGT-UKA. On the one hand, patients undergoing 3DGT-UKA need preoperative full-length CT and MRI scans, which increase medical costs and extra radiation exposure. On the other hand, specialized knowledge and special hardware and software are necessary for designing and printing an accurate $3 \mathrm{D}$ guiding template. All of these would increase the patient's economic burden. As for this study, we should also emphasize that it has some limitations. First, since the threedimensional-printed guiding template for UKA is a new technique and the exclusion criteria are very strict, there was not much sample number. Second, we only collected the data of short-term outcomes; maybe we should focus on the midterm and long-term follow-up between these two groups in the further study.

To summarize, the $3 \mathrm{D}$-printed guiding template can provide useful assistance for preoperative planning, intraoperative positioning, and osteotomy during UKA. It shortens the operation time, minimizes surgical trauma, and achieves better short-term clinical outcomes. In addition, the longterm follow-up outcomes need further research.

\section{Abbreviations}

UKA: Unicompartmental knee arthroplasty

3DGT: Three-dimensional printed patient-customized guiding template

T-UKA: Traditional UKA

HKA: Hip-knee angle

HSS: Hospital for Special Surgery

OA: Osteoarthritis

MOA: Medial compartment knee osteoarthritis

TKA: Total knee arthroplasty

3D: $\quad$ Three-dimensional

CAD: Computer-aided design.

\section{Data Availability}

The data supporting the findings is available in this manuscript, but we do not wish to share the data before it is published, because it is involved in the privacy of the patients, including age, sex, and the image of the radiology and intraoperative.

\section{Ethical Approval}

Ethical approval of the three-dimensional-printed guiding template for unicompartmental knee arthroplasty was given by the medical ethics committee of the Ethical Committee of Nanjing First Hospital with the following reference number: KY20160122-14. All of the participants agree to take part in this study; we have gained the informed consent for publication of the data from the participants.

\section{Consent}

All of the authors give permission to publish this manuscript.

\section{Conflicts of Interest}

The authors declare that they have no conflicts of interest.

\section{Authors' Contributions}

All authors were fully involved in the study and preparation of the manuscript. Dr. Fei Gu, Dr. Liangliang Li, and Huikang Zhang were mainly responsible for the collection of CT data of the patients and the 3D printing of the guide templates. Dr. Xuxiang Li and Dr. Chen Ling were mainly responsible for patient recruitment. Dr. Liming Wang, Dr. Qingqiang Yao, Dr. Fei Gu, and Dr. Liangliang Li performed the operation. Dr. Liming Wang and Dr. Qingqiang Yao were mainly responsible for designing the study and wrote the paper. Dr. Fei Gu and Dr. Liangliang Li contributed equally to this work. 


\section{Acknowledgments}

Thanks are due to Miss $\mathrm{Xu}$ and Miss Peng for their help during the collection of the patients' CT data. This study was supported by the National Key Research and Development Program of China (2018YFC1105204), National Natural Science Foundation of China (81601612, 81771985), and Key Research Program of Science \& Technology Support Program of Jiangsu Province (BE 2015613, BE 2016763).

\section{References}

[1] N. P. Kort, J. J. van Raay, J. Cheung, C. Jolink, and R. Deutman, "Analysis of Oxford medial unicompartmental knee replacement using the minimally invasive technique in patients aged 60 and above:an independent prospective series," Knee Surgery, Sports Traumatology, Arthroscopy, vol. 15, no. 11, pp. 1331-1334, 2007.

[2] F. Voss, M. B. Sheinkop, J. O. Galante, R. M. Barden, and A. G. Rosenberg, "Miller-Galante unicompartmental knee arthroplasty at 2- to 5-year follow-up evaluations," The Journal of Arthroplasty, vol. 10, no. 6, pp. 764-771, 1995.

[3] M. Chowdhry, R. S. Khakha, M. Norris, A. Kheiran, and S. K. Chauhan, "Improved survival of computer-assisted unicompartmental knee arthroplasty: 252 cases with a minimum follow-up of 5 years," The Journal of Arthroplasty, vol. 32, no. 4, pp. 1132-1136, 2017.

[4] A. Manzotti, P. Cerveri, C. Pullen, and N. Confalonieri, "Computer-assisted unicompartmental knee arthroplasty using dedicated software versus a conventional technique," International Orthopaedics, vol. 38, no. 2, pp. 457-463, 2014.

[5] R. Pailhé, J. Cognault, J. Massfelder et al., "Comparative study of computer-assisted total knee arthroplasty after opening wedge osteotomy versus after unicompartmental arthroplasty," The bone \& joint journal, vol. 98, no. 12, pp. 1620$1624,2017$.

[6] D. K. Bae and S. J. Song, "Computer assisted navigation in knee arthroplasty," Clinics in Orthopedic Surgery, vol. 3, no. 4, pp. 259-267, 2011.

[7] D. Saragaglia, J. Massfelder, R. Refaie et al., "Computerassisted total knee replacement after medial opening wedge high tibial osteotomy: medium-term results in a series of ninety cases," International Orthopaedics, vol. 40, no. 1, pp. 35-40, 2017.

[8] P. Hernigou and G. Deschamps, "Alignment influences wear in the knee after medial unicompartmental arthroplasty," Clinical Orthopaedics and Related Research, vol. 423, pp. 161-165, 2004.

[9] P. Hernigou and G. Deschamps, "Posterior slope of the tibial implant and the outcome of unicompartmental knee arthroplasty," The Journal of Bone and Joint Surgery. American Volume, vol. 86, no. 3, pp. 506-511, 2004.

[10] N. P. Kort, J. J. van Raay, and B. J. Thomassen, “Alignment of the femoral component in a mobile-bearing unicompartmental knee arthroplasty: a study in 10 cadaver femora," The Knee, vol. 14, no. 4, pp. 280-283, 2007.

[11] T. Walker, T. Gotterbarm, T. Bruckner, C. Merle, and M. R. Streit, "Total versus unicompartmental knee replacement for isolated lateral osteoarthritis: a matched-pairs study," International Orthopaedics, vol. 38, no. 11, pp. 2259-2264, 2014.
[12] B. Bordini, S. Stea, S. Falcioni, C. Ancarani, and A. Toni, "Unicompartmental knee arthroplasty," The Knee, vol. 21, no. 6, pp. 1275-1279, 2014.

[13] O. R. Kwon, K. T. Kang, J. Son, D. S. Suh, C. Baek, and Y. G. Koh, "Importance of joint line preservation in unicompartmental knee arthroplasty: finite element analysis," Journal of Orthopaedic Research, vol. 35, no. 2, pp. 347-352, 2017.

[14] B. Ma, W. Long, J. F. Rudan, and R. E. Ellis, “Three-dimensional analysis of alignment error in using femoral intramedullary guides in unicompartmental knee arthroplasty," The Journal of arthroplasty, vol. 21, no. 2, pp. 271-278, 2006.

[15] A. Baldini and P. Adravanti, "Less invasive TKA," Clinical Orthopaedics and Related Research, vol. 466, no. 11, pp. 2694-2700, 2008.

[16] V. De Santis, A. Burrofato, R. D’Apolito et al., "Evaluation of accuracy of bone cuts and implant positioning in total knee arthroplasty using patient specific instrumentation," Journal of Biological Regulators \& Homeostatic Agents, vol. 31, 4 suppl 1, pp. 51-60, 2017.

[17] J. Y. Jenny and C. Boeri, "Accuracy of implantation of a unicompartmental total knee arthroplasty with 2 different instrumentations," The Journal of Arthroplasty, vol. 17, no. 8, pp. 1016-1020, 2002.

[18] G. Keene, D. Simpson, and Y. Kalairajah, "Limb alignment in computer-assisted minimally-invasive unicompartmental knee replacement," Journal of Bone and Joint Surgery. British Volume (London), vol. 88, pp. 44-48, 2006.

[19] R. A. Berger, R. M. Meneghini, J. J. Jacobs et al., "Results of unicompartmental knee arthroplasty at a follow-up of tenyears follow-up," Journal of Bone and Joint Surgery, vol. 87, pp. 999-1006, 2005.

[20] J. Newman, R. V. Pydisetty, and C. Ackroyd, "Unicompartmental or total knee replacement: the 15 -year results of a prospective randomised controlled trial," Journal of Bone and Joint Surgery. British Volume (London), vol. 91, pp. 52-57, 2009. 